\title{
The efficacy of a non-leaching antibacterial central venous catheter - a prospective, randomized, double-blind study
}

\author{
Ivo Krikava ${ }^{a}$, Martin Kolar ${ }^{b}$, Barbora Garajova ${ }^{c}$, Tomas Balik $^{\mathrm{d}}$, Alena Sevcikova ${ }^{e}$, Ingolf Roschke', Pavel Sevcik ${ }^{\mathrm{g}}$
}

\begin{abstract}
Background. Antimicrobial coatings of central venous catheters (CVC) have the potential to reduce the risk of infectious complications. The aim of this study was to examine the efficacy of a catheter with a non-leaching antimicrobial coating against catheter colonization and bloodstream infections (BSI).

Methods. The study was conducted in two centers using a prospective, randomized, double-blind and controlled design ( 680 intensive care patients; a protective CVC (Certofix ${ }^{\circledast}$ protect) or a standard CVC (Certofix ${ }^{\odot}$ ). Primary objectives were the rates of catheter colonization and BSI in the two groups. Other baseline demographics, APACHE II score, insertion site, location of CVC placement (ICU or theatre), indwelling time and length of ICU stay were comparable for both groups.

Results. While the rate of catheter colonization between the coated and uncoated CVC ( $17.4 \%$ vs. $18.7 \%, P=0.7477)$ and the rate of microbiologically confirmed catheter associated infections were similar ( $1.4 \%$ vs. $1.9 \%, P=0.7521)$, the coated CVC showed a significantly lower incidence of BSI $(2.0 \%$ vs. $6.5 \%, P=0.0081)$ and a significantly lower mean incidence of BSI per 1000 catheter days ( 3.2 vs. 8.3, $P=0.0356$ ).

Conclusion. The non-leaching antibacterial coating of the protective catheter was effective in reducing the incidence of $\mathrm{BSI}$ but not the rate of catheter colonization. However, the incidence of $\mathrm{BSI}$ is a better surrogate marker for the risk of developing clinical signs of infection suggesting that use of the non-leaching protective catheter is effective in this regard.
\end{abstract}

Trial number: ClinicalTrials.gov (ID: NCT00555282), https://clinicaltrials.gov/show/NCT00555282

Key words: antimicrobial, CVC, non-leaching, catheter-related bloodstream infections

Received: February 7, 2019; Accepted with revision: May 15, 2019; Available online: May 28, 2019

https://doi.org/10.5507/bp.2019.022

(c) 2020 The Authors; https://creativecommons.org/licenses/by/4.0/

${ }^{a}$ Department of Pain Treatment, Department of Paediatric Anaesthesiology and Resuscitation, University Hospital Brno and Department of Paediatric Anaesthesiology and Resuscitation, Faculty of Medicine, Masaryk University, Brno, Czech Republic

${ }^{b}$ Department of Anesthesiology and Critical Care, University Hospital Kralovske Vinohrady, Prague, and Department of Anaesthesiology and Resuscitation, 3rd Faculty of Medicine, Charles University, Prague, Czech Republic

'Department of Surgery, University Hospital Brno, Brno, Czech Republic

${ }^{d}$ Department of Anesthesiology and Critical Care, University Hospital Kralovske Vinohrady, Prague, Czech Republic

e Department of Clinical Microbiology, University Hospital Brno, Brno and Department of Laboratory Methods, Faculty of Medicine, Masaryk University, Brno, Czech Republic

${ }^{f} \mathrm{Dr}$. Roschke GMBH, Cologne, Germany

${ }^{9}$ Depatment of Anaesthesiology and Resuscitation, University Hospital Ostrava and Department of Intensive Medicine and Forensic Studies, Faculty of Medicine, University of Ostrava, Ostrava, Czech Republic

Corresponding author: Pavel Sevcik, e-mail:pavel.sevcik@fno.cz

\section{BACKGROUND}

Central venous catheters (CVC) have become an indispensable part of patient management ${ }^{1,2}$. However, the use of CVC is fraught with the risk of infectious complications particularly in critically ill patients ${ }^{1,3}$. Catheterrelated bloodstream infections (CRBSI) are the most serious cause of health-care associated bloodstream infections with a stated incidence of three to eight percent of inserted catheters. They are associated with increased morbidity, duration of stay in hospital and excess health care costs. Estimated attributable mortality is up to 17 percent $^{1,47}$.

Much effort has been invested in prevention strategies to decrease catheter-related colonization and CRBSI such as the use of a maximum sterile barrier technique, chlorhexidine-containing medical devices, educational programs for health care workers, comprehensive prevention programs, catheter hubs containing iodinated alcohol, and use of biomaterials that inhibit microorganism growth and adhesion ${ }^{4,7}$.

Catheters with antimicrobial or antiseptic impregnations coated onto or incorporated into the catheter polymer have been developed ${ }^{5,8,9}$. Currently several antimicrobial CVC are commercially available. The main four types of impregnated or coated CVC are leaching systems whose modes of action are based on drug release ${ }^{5,9}$. Various studies have demonstrated beneficial effects of these coatings in reducing colonization and CRBSI (ref.'-12). However, these catheters have some disadvantages, e.g. limited time of drug release, risk of toxic or anaphylactic reactions, and discussed risk of bacterial 
resistance against antibiotics ${ }^{5,9,13,14}$. Therefore it is recommended to limit their use to intensive care ${ }^{4}$. The advantage of coated CVC in comparison with silver-impregnated CVC could be lower cost on the market.

A new type of coated catheter with a non-leaching antibacterial coating was recently developed (Certofix ${ }^{\circledR}$ protect, B.Braun). With this catheter the active agent polyhexanide cannot be flushed out from the catheter. The repelling antimicrobial property of this non-leaching catheter is based on a purely physical effect. Thereby, polyhexanide is chemically bonded to the inner and outer surface of polyurethane catheter material resulting in long-lasting polarization of the catheter surface. The bactericidal effect is supposed to occur through the positively charged catheter surface which physically destroys the lipid bilayer surface of bacteria. (Information provided by B.Braun). Recently, an in vitro study showed a significant inhibitory effect on biofilm formation (for Staphylococcus aureus, S. epidermidis, Pseudomonas aeruginosa, Acinetobacter baumannii, Klebsiella pneumoniae, and Candida albicans) of Certofix ${ }^{\circledR}$ protect in comparison to non-coated catheters ${ }^{15}$.

\section{AIM}

The aim of this clinical study was to examine the safety and efficacy of Certofix ${ }^{\circledR}$ protect in respect to the rate of catheter colonization and the risk of bloodstream infections (BSI) in the setting of multidisciplinary intensive care units (ICU).

\section{METHODS}

\section{Study Design}

The study was designed as a prospective, controlled, double-blind, randomized post-approval medical device study in two centers in the Czech Republic (University Hospital Brno, University Hospital Kralovske Vinohrady, Prague). It was planned with an adaptive design (see interim analysis). The study was approved by ethic committees in both centers.

\section{Number of patients}

The originally planned number of patients was 480 (240 patients per group). After interim analysis, the number of patients was adapted to 680 (340 patients per group). (see interim analysis)

\section{Study population}

All patients aged 18 years or older with a probable stay in ICU after CVC placement were eligible. They were included in case of a first or second central venous catheterization during actual hospital stay and an anticipated CVC indwelling period in the subclavian or jugular vein of more than three days. Written informed consent by the patient or the attending physician prior to participation was required. In case of the latter, written informed consent by the patient was to be obtained after recovery if possible. Exclusion criteria included (1) placement in the femoral vein, (2) inflammation of the skin at the site of puncture prior to the puncture, (3) known hypersensitivity to any of the components (i.e. polyhexanide or related substance such as chlorhexidine), (4) emergency insertion of the catheter in the field, and (5) participation in another clinical trial.

\section{Study catheter}

The study catheter (Certofix ${ }^{\circledR}$ protect, B.Braun) was a non-leaching antibacterial CVC while the control catheter (Certofix ${ }^{\circledR}$, B.Braun) was a non-coated CVC. Both catheters are CE-marked and are made of polyurethane.

\section{Blinding and Randomization}

Study and control catheters and their packaging looked identical and could only be identified by study number.

Patients received a sequential number in accordance with their order of inclusion into the study. They were assigned to either of the two devices by a coded randomization list. Randomization took place immediately before catheter placement. Neither the clinical investigator nor the patients were informed of the type of used catheter.

\section{Documentation and Visit Schedule}

Each patient's course of therapy was examined. The physician documented each visit on a standardised case report form. During the initial visit, patients were checked for study eligibility and written consent for study participation was obtained. The catheters were handled according to standardised catheter care guidelines (e. g. sterile barrier concept, dressing change and routine cap change, flush and heparinisation, no special connectors or valves were used). The most common disinfectants were iodine and alcohol based skin antiseptics.

The catheter insertion site was examined daily. After removal of the catheter a microbial examination of the catheter was performed. The study was terminated at the removal of the last investigational catheter.

\section{Outcome Measures}

The original primary endpoint of the study was the incidence of 'catheter tip colonization'. After interim analysis, the primary outcome was changed to 'incidence of bloodstream infections' (BSI) which was a secondary endpoint before (see interim analysis).

At the beginning of the study in 2005, no definition of catheter-related bloodstream infection from the CDC or IDSA was available. Catheter colonisation was evaluated by establishing cultures: after catheter removal $5 \mathrm{~cm}$ of intravascular segment was cut and placed in a sterile container or culture medium. The quantificative sonification method was used to determine the colonisation of the catheter tip. In the presence of clinical signs of bloodstream infection or sepsis, blood cultures were drawn from the catheter and peripheral vein. A bloodstream infection was defined as a recognised pathogen in the blood, when the pathogen was not related to an infection at another site, or when there were clinical signs of 
infection (fever, chills, or hypotension - systolic pressure $\leq 90 \mathrm{~mm} \mathrm{Hg}$ ).

Clinical sepsis was diagnosed when the patient had fever, hypotension, or oliguria and when either there had been no blood culture or no microorganism had been isolated. Additional conditions were that there was no apparent infection at another site or that a physician had initiated appropriate antimicrobial therapy. These definitions were valid at the beginning of the study.

Concomitant study parameters comprised baseline patient data, data regarding the CVC, clinical therapy, APACHE II- and TISS-scores, invasive procedures while the central line was in place, basic laboratory investigations (CRP, leukocytes), and length of ICU stay.

\section{Statistical analyses}

All analyses were performed with the intention-totreat (ITT) population (all patients who were randomized and received treatment) and the per-protocol (PP) population (all patients with a catheter indwelling period of more than three days). Primary parameters were analyzed with confirmative statistical tests and the usual descriptive statistical methods. On all secondary parameters data analysis was carried out. All statistical tests were performed two-sided with a significance level of $\alpha=0.05$. Fisher's exact test was used for two times two tables while for all other contingency tables the $\chi^{2}$-Test was used. For the analyses of continuous parameters, the groups were compared with the t-test.

\section{Interim analysis}

After inclusion of 241 patients (half of the originally planned sample size) a planned interim analysis was performed. The analysis of catheter colonization showed no difference between standard CVC and coated CVC (20.5\% vs. $20.8 \%$; $P=0.9632$ ) implying discontinuation of the study in the first instance. The results of the interim analysis showed no statistically significant difference between the groups in the incidence of BSI being $4.0 \%$ in the Certofix ${ }^{\circledR}$ protect group and $9.7 \%$ in the Certofix ${ }^{\circledR}$ standard group $(P=0.1026)$. Based on the data of the interim analysis, a significant difference regarding the incidence of BSI was to be expected if the study were to be completed with a new sample size.

The rationale for the increase of sample size was based on the results of Pearson Test for the adapted primary objective showing a difference between the two catheter groups for the incidence of BSI ( $4.0 \%$ for coated CVC vs. $9.7 \%$ for uncoated CVC) with a $P$ value of 0.1026 . According to the adaptive design of Bauer and Köhne, the two sided $\alpha$-value for the second stage of the study was 0.0848 (ref. ${ }^{16,17}$ ). Due to practical reasons and speed of recruitment in the two study centers, a sample size of 220 patients per group for the second stage was regarded most adequate, corresponding to a power of $74 \%$. Therefore a total of 680 patients were included in the study.

To avoid bias neither investigators, staff nor the study monitor were informed about the results of the interim analysis.

\section{FINDINGS}

The duration of study was about 40 months from December 2005 to April 2009. 680 patients were included in the study. Due to loss to follow-up in five patients and femoral catheter access in one patient, the ITT- population included 674 patients. As 58 patients had a catheter indwelling time of less than three days, the PP-population included 616 patients (304 patients in the coated and 312 in the uncoated CVC group). The difference between ITTand PP-population was not significant. All results pertain to the PP-population (Fig. 1).

There were no differences in baseline demographic data or clinical characteristics between the two groups. The mean APACHE II score was comparable (10.6 \pm 8.7 and $11.3 \pm 8.5$ in the coated and uncoated group, $P=0.33$ ) (Table 1).

Table 1. Patient baseline data in the Certofix ${ }^{\circledR}$ protect and Certofix ${ }^{\circledR}$ standard group.

\begin{tabular}{llll}
\hline & Certofix $^{\circledR}$ protect & Certofix $^{\circledR}$ standard & $P$ \\
\hline Sex male & $58.5 \%$ & $62.8 \%$ & 0.2846 \\
Age (mean) & $60.7 \pm 13.9$ years & $60.7 \pm 15.6$ years & 0.9704 \\
BMI (mean) & $26.1 \pm 4.7 \mathrm{~kg} / \mathrm{m}^{2}$ & $26.6 \pm 5.7 \mathrm{~kg} / \mathrm{m}^{2}$ & 0.2192 \\
APACHE II, mean & $10.6 \pm 8.7$ & $11.3 \pm 8.5$ & 0.3300 \\
\hline
\end{tabular}

Table 2. Catheter parameters related to placement in the Certofix ${ }^{\circledR}$ protect and Certofix ${ }^{\circledR}$ standard group.

\begin{tabular}{llccc}
\hline & & Certofix $^{\circledR}$ & Certofix $^{\circledR}$ & $P$ \\
protect $\left.^{\circledR} \%\right)$ & standard (\%) & \\
\hline Access & v. jugularis & 19.1 & 16.7 & 0.4623 \\
& v. subclavia & 80.9 & 84.3 & 0.2885 \\
Placement in & operating room & 47.0 & 46.8 & 1.00 \\
& ICU & 53.0 & 52.9 & 1.00 \\
& intermediate care & 0 cases & 2 cases & 0.1540 \\
Complications & & 13.9 & 18.3 & 0.0663 \\
Of these handling problems & 4.8 & 17.5 & \\
\hline
\end{tabular}


More than $80 \%$ of the catheters could be placed without complications. If problems occurred, they could mostly be related to the puncture. There was a trend towards more handling problems during catheter placement in the uncoated CVC group ( $17.5 \%$ vs. $4.8 \%$ in the coated group; $P=0.0663)$. In half of patients in both groups, catheter fixation was conducted according to study protocol. For the other half of patients, an equivalent dressing was used (Table 2).

The catheter indwelling time was at least three days up to approximately 21 days (maximum in one case was 27 days) with a mean indwelling period of $7.3 \pm 2.9$ vs. 7.73 .2 in the coated vs. the uncoated group. The average length of stay in ICU was $8.5 \pm 12.3$ vs. $8.8 \pm 8.2$ days in the coated vs. the uncoated group $(P=0.7436)$. The number of invasive procedures (artificial respiration, surgery, number of drains, urological catheter, and others) was high in both groups but no significant differences in frequency and duration of the procedures were observed $(P>0.05)$.

After removal, the majority of catheter tips were microbiologically investigated in both groups $(95.1 \%$ and $96.2 \%$ in the coated and uncoated CVC group). Microbial findings did not differ between the groups and displayed catheter colonization rates of $17.4 \%$ and $18.7 \%$ for the coated and uncoated CVC $(P=0.7477)$, respectively (Table 3).

The rate of BSI was significantly lower in the coated than in the uncoated CVC group $(2.0 \%$ vs. $6.5 \%$; $P=0.0081$ ) (Fig. 2). Also the BSI rate per 1000 catheter days was significantly lower in the coated CVC group (mean 3.2 vs. $8.3 ; P=0.0356$ ). In contrast, the rate of clinical sepsis did not differ between the groups ( $7.0 \%$ vs. $6.5 \%$ in the coated vs. the uncoated group; $P=0.8720$ ) nor did

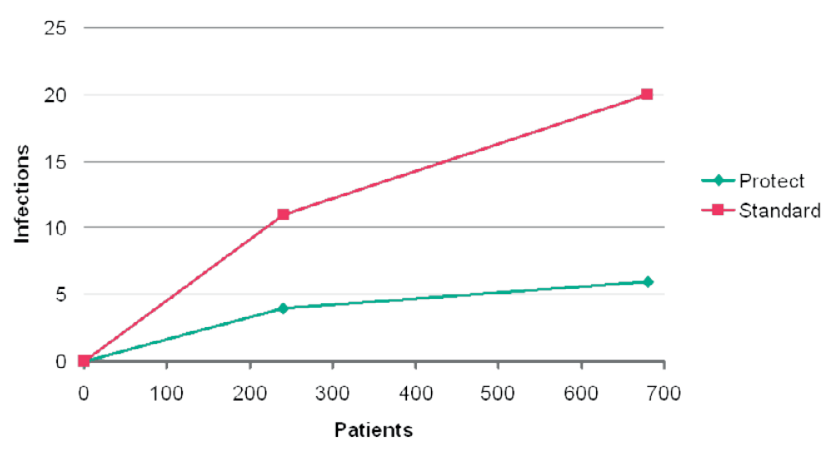

Fig. 1. Flowchart of patient recruitment (in number of patients) and population arrangement.

the frequency of microbiologically confirmed catheter associated infections ( $1.3 \%$ vs. $1.9 \%$ in the coated vs. the uncoated group; $P=0.7521$ ) (Table 3 ). According to BSI and positive blood cultures no cases of infectious endocarditis (or valvular vegetations) were recorded in any patients.

The pathogens of catheter colonisation and catheter-related bloodstream infections were coag. neg Staphylococcus, Staphylococcus aureus, Enterococcus faecalis, MRSA, Pseudomonas aeruginosa, Candidia albicans, Klebsiella pneumonia, Escherichia coli, Klebsiella oxytoca, Enterobacter cloacae, Bacillus cereus and Acinetobacter baumanii. There were no clinically differences in the predominant pathogens observed between the two types of catheters. Moreover, there were no significant differences between the catheters with respect to catheter care and dressing change observed. The most frequently used disinfectant was Iodisol ${ }^{\circledR}$ (Povidone-iodine solution in 95\% ethanol).

Table 3. Infection parameters in the Certofix ${ }^{\circledR}$ protect and Certofix ${ }^{\circledR}$ standard group.

\begin{tabular}{lccc}
\hline & $\begin{array}{c}\text { Certofix } \\
\text { protect }\end{array}$ & $\begin{array}{c}\text { Certofix }^{\circledR} \\
\text { standard }\end{array}$ & $P$ \\
\hline Tip colonization & $17.4 \%$ & $18.7 \%$ & 0.7477 \\
Bloodstream infections (BSI) & $2.0 \%$ & $6.5 \%$ & 0.0081 \\
BSI per 1000 catheter days (mean) & $3.2 \pm 24.5$ & $8.3 \pm 34.4$ & 0.0356 \\
Clinical sepsis & $7.0 \%$ & $6.5 \%$ & 0.8720 \\
Microbiologically confirmed catheter & $1.3 \%$ & $1.9 \%$ & 0.7521 \\
associated infection & & & \\
\hline
\end{tabular}

Table 4. Differences in the incidences of catheter tip colonization and bloodstream infection, APACHE II score, insertion sites and placement locations and antibiotics between the two study centers.

\begin{tabular}{lccr}
\hline & Prague & Brno & $P$ \\
\hline Tip colonization & $14.4 \%$ & $21.8 \%$ & 0.0240 \\
Bloodstream infections & $6.4 \%$ & $2.2 \%$ & 0.0148 \\
Mean APACHE II score & $13.3 \pm 10.8$ & $8.6 \pm 4.8$ & $<0.0001$ \\
Access v. jugularis & $5.6 \%$ & $29.7 \%$ & $<0.0001$ \\
Placement in operating room & $0.7 \%$ & $91.7 \%$ & $<0.0001$ \\
Placement in ICU & $99.3 \%$ & $8.0 \%$ & $<0.0001$ \\
Administration of antibiotics & $84.8 \%$ & $96.5 \%$ & $<0.0001$ \\
Mean duration of antibiotics & 8.0 days & 2.3 days & 0.0005 \\
\hline
\end{tabular}




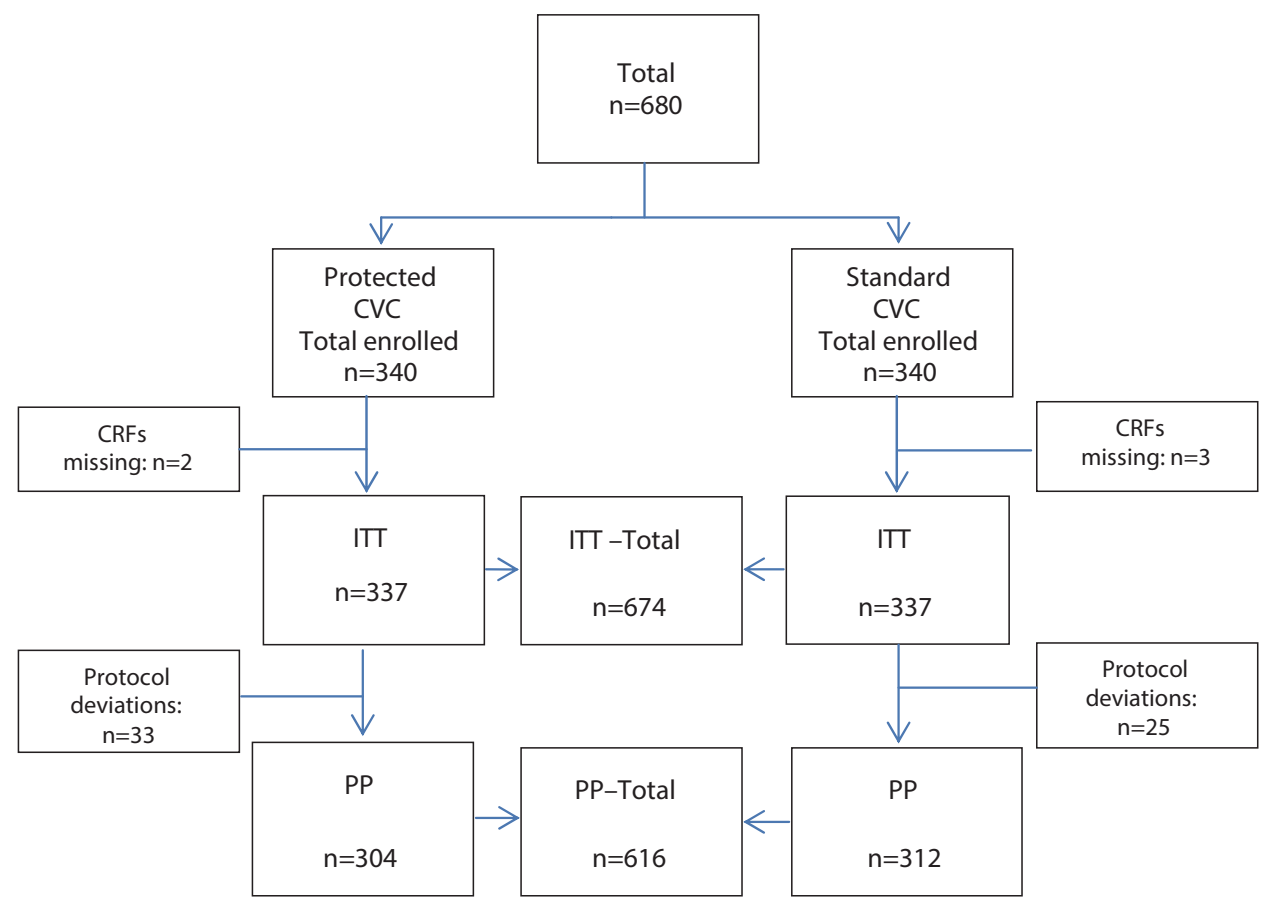

Fig. 2. Relative number of infections in the Certofix ${ }^{\circledR}$ protect and the Certofix ${ }^{\circledR}$ standard group at time of interim analysis and end of study.

The antimicrobial therapy was, in all cases therapeutic (not prophylactic) and the empirical treatment was targeted to a few days according to microbiological findings. In the non-coated group the frequency of administering antibiotics was significantly higher (93.3\% vs. $88.2 \%$ in the coated group; $P=0.0364)$. However, the duration of their application was not significantly higher in the uncoated group ( 5.8 vs. 4.0 days in the coated group; $P=0.2051$ ).

In total, 129 adverse events (AEs) were documented from 81 PP-patients: 48 with the coated CVC (Certofix ${ }^{\circledR}$ protect) and 81 with the uncoated standard catheter (Certofix $\left.{ }^{\circledR}\right)$. Neither in the PP- nor the ITT-population were significant differences between the two device groups found $(P>0.05)$. The occurrence of an infection was the most frequent event $(50.0 \%$ of the AEs in the protected catheter group were classified as infections/infestations as well as $48.2 \%$ in the standard catheter group).

The intensity of more than half of the AEs was classified as 'moderate' ( $54.2 \%$ vs. $64.2 \%$ in the coated vs. uncoated group), only about $10 \%$ as 'slight' ( $n=6$ vs. $\mathrm{n}=7$ in the coated vs. uncoated group), and a third as 'severe' (33.3\% vs. $27.2 \%$ in the coated vs. uncoated group). A fourth of the AEs were classified as 'serious'. Of the 30 serious AEs documented in 27 patients, twelve occurred with the coated catheter and 18 with the noncoated catheter.

At the end of the study, only six cases with present symptoms were documented. In the PP-population 16 non-related deaths occurred ( $n=6$ in the coated and $\mathrm{n}=10$ in the uncoated group).

\section{DISCUSSION}

Certofix ${ }^{\circledR}$ protect showed a significant decrease in BSI (2.0\% vs. $6.5 \%)$ and BSI per 1000 catheter days (3.2 vs. 8.3). A significant decrease in the percentage of patients receiving antibiotics (88.2\% vs. $93.3 \%)$ was also detectable for the benefit of the protected CVC. Those numbers reveal the favorable situation of Certofix ${ }^{\circledR}$ protect with regard to infection parameters and reflect the antimicrobial effect of the new polymer surface of the coated catheter. Study results compare favorably with reported CVC infection rates of $1.6 \%$ to $10 \%$ and incidences of CR-BSI ranging from 1.8 to 5.2 per 1000 catheter days in other studies ${ }^{6,17-19}$. However, in this study the rate of clinical sepsis did not differ between the groups (7.0\% vs. $6.5 \%$ in the coated vs. uncoated group; $P=0.8720)$.

In a review by Frasca two studies evaluated catheters coated on both their external and internal surfaces and both provided comparable results ${ }^{4}$. In other studies with coated catheters inconsistent data is reported. While some did not reveal any differences in catheter colonization for different catheter types either, others observed beneficial effects of different coatings in reducing colonization $^{9-12,14,20-22}$.

The rate of catheter tip colonisation in both groups was comparable and naturally higher than the blood stream infection rate which differed between groups. This finding implies that the direct antimicrobial effect of catheter coating could be probably insignificant, whereas releasing of bacteria's or parts of biofilm from colonisated catheters to the bloodstream seems to be clinically more significant and more affected by catheter coating. This effect could be caused by difference in (chemical 
and physical) features of outer and inner surface in both catheter groups (charge, hydrophilicity).

It should be noted that the 'incidence of BSI' is a clinically relevant parameter influencing the patients' outcome whereas 'incidence of catheter tip colonization' is only an artificial measure. Equally, it is a more informative parameter with regards to the risk of infection. In contrast, catheter colonization may be influenced by differing catheter handling and varying laboratory practice which cannot easily be standardized in a clinical study.

The vena subclavia was the most frequent access site with more than $80 \%$ for both types of catheter. The preference for the subclavian route is consistent with current data from literature that mostly states a slightly higher infection risk for jugular and much higher for femoral vein access $^{4,19,23,24}$. Infection rates of 3.8 per 1000 catheter days in subclavian, 6.1 in jugular, and 15.7 in femoral vein catheterization, and infection rates of 5.4 per 1000 catheter days for subclavian and 10.2 for jugular catheterization in ICU have been reported ${ }^{18}$. In one study no statistical difference in the rate of CRBSI between the subclavian and jugular access route was seen ${ }^{6}$.

In this study in each group about half of the catheters were placed in the operating room and the other half in the ICU. Therefore location of placement should have had no effect on the results. Differences in infection rates between catheter placement in the ICU and the operating theatre have been observed in the literature. One study reported that CVCs inserted in the ICU are associated with much higher infection rates than in the operating room (9.4\% and $1.4 \%$, respectively) (ref. $\left.{ }^{6}\right)$. In another study, however, no difference in CRBSI rates between ICU and operating room was noted ${ }^{17}$.

The coated catheter can be considered safe. Of the 129 AEs that were seen in 81 PP-patients, more occurred in the standard group, most were classified as moderate and not device related.

Efficacy of coated CVC was proven in many studies and use of similar catheters is recommended in current guidelines especially when the catheter is in place for more than 5 days $^{25}$.

\section{CONCLUSION}

In summary, the non-leaching antibacterial catheter of Certofix ${ }^{\circledR}$ protect can be regarded as effective and safe. The coated CVC showed a significantly lower incidence of BSI and a lower mean incidence of BSI per 1000 catheter days. All documented safety parameter showed safe handling.

\section{ABBREVIATIONS}

AEs, Adverse events; BSI, Bloodstream infections; CDC, Centers for Disease Control and Prevention; CRBSI (CR-BSI), Catheter-related bloodstream infection; CRP, C-reactive Protein ; CVC, Central venous catheter;
ICU, Intensive care unit ; ITT, Intention-to-treat; IDSA, Infectious Diseases Society of America; PP, Per-protocol.

\section{Ethics approval and consent to participate}

Before initiating the study, the investigator had obtained written and dated approval/favourable opinion from the relevant Institutional Review Board/Independent Ethics Committee for the study protocol (and any amendments), written informed consent form, consent form updates, patient recruitment procedures (e.g. advertisements), and any other written information to be provided to patients. Approval was indicated in writing with reference to the final protocol number and date. Details of the Independent Ethics Committees constitution were included in the Trial Master File. During the study, the investigator provided to the Review Board/Independent Ethics Committee all documents that were subject to review.

Ethic Committees: Ethics Committee for MultiCentric Clinical Trial of the University Hospital Motol, reference number: EK/32/2005, approved: 13.7.2005, reference number (multicentric ethic committee): EK$1135 / 05$, approved 19.10.2005

Ethics Committee of the University Hospital Brno, reference number: 063/05MEK, approved: 16.11.2005

Acknowledgement: This study was funded by B. Braun Melsungen AG, Melsungen, Germany.

Author contributions: IK, MK, BG, TB, and AS designed and conducted the study. IK and PS commented the drafts of the paper. IR wrote and designed the manuscript. All authors read and approved the final manuscript.

Conflict of interest statement: The authors declare no competing interest. Ingolf Roschke is the managing director of Dr. Roschke GMBH

Availability of data and material: The datasets generated during the current study are not publicly available due to the data protection of the patients.

\section{REFERENCES}

1. Maki DG, Kluger DM, Crnich CJ. The risk of bloodstream infection in adults with different intravascular devices: a systematic review of 200 published prospective studies. Mayo Clin Proc 2006;81:1159-71.

2. Teichgräber UK, Gebauer B, Benter T, Wagner J. Long-term central venous lines and their complications. Rofo 2004;176: 944-52.

3. Simon A, Ammann RA, Bode U, Fleischhack G, Wenchel HM, Schwamborn D, Gravou C, Schlegel PG, Rutkowski S, Dannenberg C, Körholz D, Laws HJ, Kramer MH. Healthcareassociated infections in pediatric patients: results of a prospective study from university hospitals in Germany and Switzerland. BMC Infectious Diseases 2008;8:70.

4. Frasca D, Dahyot-Fizelier C, Mimoz O. Prevention of central venous catheter-related infection in the intensive care unit. Critical Care 2010;14:212.

5. Byrnes MC, Coopersmith CM. Prevention of catheter-related bloodstream infection. Curr Opin Crit Care 2007;13:411-5.

6. Tan CC, Zanariah Y, Med M, Lim KI, Balan S. Central venous catheterrelated bloodstream infections: incidence and an analysis of risk factors. Med J Malaysia 2007;62:370-4.

7. Yousif A, Jamal MA, Raad I. Biofilm-based central line-associated bloodstream infections. Adv Exp Med Biol. 2015;830:157-79. 
8. Elliot P. Prevention of intravascular catheter-related infections. In: Hamilton $\mathrm{H}$, Bodenham AR, editors. Central venous catheters. Chichester: Wiley-Blackwell; 2009. p. 210-2.

9. Gilbert RE, Harden M. Effectiveness of impregnated central venous catheters for catheter related bloodstream infection: a systematic review. Curr Opin Infect Dis 2008;21:235-45.

10. Wang $H$, Huang T, Jing J, Jin J, Wang P, Yang M, Cui W, Zheng Y, Shen $\mathrm{H}$. Effectiveness of different central venous catheters for catheterrelated infections: a network meta-analysis. J Hosp Infect 2010;76:111.

11. Ramritu P, Halton $K$, Collignon P, Cook D, Fraenkel D, Battistutta $D$ Whitby $M$, Graves N. A systematic review comparing the relative ef fectiveness of antimicrobial-coated catheters in intensive care units. Am J Infect Control 2008;36:104-17.

12. Wang $H$, Tong $H$, Liu $H$, Wang $Y$, Wang $R$, Gao $H$, Yu P, Lv Y, Chen S, Wang G, Liu M, Li Y, Yu K, Wang C. Effectiveness of antimicrobialcoated central venous catheters for preventing catheter-related blood-stream infections with the implementation of bundles: a systematic review and network meta-analysis. Ann Intensive Care. 2018;8(1):71.

13. Hockenhull JC, Dwan K, Boland A, Smith G, Bagust A, Dündar Y, Gamble C, McLeod C, Walley T, Dickson R. The clinical effectiveness and cost-effectiveness of central venous catheters treated with antiinfective agents in preventing bloodstream infections: a systematic review and economic evaluation. Health Technology Assessment 2008;12:1-154.

14. Chong HY, Lai NM, Apisarnthanarak A, Chaiyakunapruk N. Comparative Efficacy of Antimicrobial Central Venous Catheters in Reducing Catheter-Related Bloodstream Infections in Adults: Abridged Cochrane Systematic Review and Network MetaAnalysis. 2017;64(suppl_2):131-40.

15. Richards GA, Brink AJ, McIntosh R, Steel HC, Cockeran R. Investigation of biofilm formation on a charged intravenous catheter relative to that on a similar but uncharged catheter. Med Devices (Auckl) 2014;7:219-24

16. Bauer P, Köhne K. Evaluation of Experiments with Adaptive Interim Analysis: Biometrics 1994;50(4):1029-41.
17. Gowardman JR, Robertson IK, Parkes S, Rickard CM. Influence of insertion site on central venous catheter colonization and bloodstream infection rates. Intensive Care Med 2008;34:1038-45.

18. Nagashima G, Kikuchi T, Tsuyuzaki H, Kawano R, Tanaka H, Nemoto $\mathrm{H}$, Taguchi K, Ugajin K. To reduce catheter-related bloodstream infections: is the subclavian route better than the jugular route for central venous catheterization? J Infect Chemother 2006;12:363-5.

19. van der Kooi TII, Wille JC, van Benthem BHB. Catheter application, insertion vein and length of ICU stay prior to insertion affect the risk of catheter-related bloodstream infection. J Hosp Infect 2012;80:23844.

20. Camargo LF, Marra AR, Büchele GL, Sogayar AM, Cal RG, de Sousa $J M$, Silva E, Knobel E, Edmond MB. Double-lumen central venous catheters impregnated with chlorhexidine and silver sulfadiazine to prevent catheter colonisation in the intensive care unit setting: a prospective randomised study. J Hosp Infect 2009;72:227-33.

21. Kalfon $P$, de Vaumas $C$, Samba D, Boulet $E$, Lefrant JY, Eyraud D, Lherm T, Santoli F, Naija W, Riou B. Comparison of silver-impregnated with standard multi-lumen central venous catheters in critically ill patients. Crit Care Med 2007;35:1032-9.

22. Darouiche RO, Berger DH, Khardori N, Robertson CS, Wall MJ Jr, Metzler MH, Shah S, Mansouri MD, Cerra-Stewart C, Versalovic J, Reardon MJ, Raad II. Comparison of antimicrobial impregnation with tunneling of long-term central venous catheters: a randomized controlled trial. Ann Surg 2005;242:193-200.

23. O'Grady NP, Alexander M, Burns LA, Dellinger EP, Garland J, Heard SO, Lipsett PA, Masur H, Mermel LA, Pearson ML, Raad II, Randolph AG, Rupp ME, Saint S, Healthcare Infection Control Practices Advisory Committee (HICPAC) (2011). Guidelines for the prevention of intravascular catheter-related infections. Clin Infect Dis. 2011;52(9):e16293

24. Charalambous CC, Swobody SM, Dick J, PerlT, Lispett PA. Risk factors and clinical impact of central line infections in the surgical intensive care unit. Arch Surg 1998;133(11):1241-6.

25. Lorente L. Antimicrobial-impregnated catheters for the prevention of catheter-related bloodstream infections. World J Crit Care Med 2016;5(2):137-42 